\title{
ON THE (UN)DECIDABILITY OF A NEAR-UNANIMITY TERM
}

\author{
MIKLÓS MARÓTI
}

\begin{abstract}
We investigate the near-unanimity problem: given a finite algebra, decide if it has a near-unanimity term of finite arity. We prove that it is undecidable of a finite algebra if it has a partial near-unanimity term on its underlying set excluding two fixed elements. On the other hand, based on Rosenberg's characterization of maximal clones, we present partial results towards proving the decidability of the general problem.
\end{abstract}

\section{INTRODUCTION}

We call a term $t$ of an algebra $\mathbf{A}$ a near-unanimity term if it satisfies the following identities:

$$
t(y, x, \ldots, x) \approx t(x, y, x, \ldots, x) \approx \cdots \approx t(x, \ldots, x, y) \approx x .
$$

For brevity, we sometimes write NU-term instead of near-unanimity term. We investigate the near-unanimity problem: given a finite algebra, decide if it has a near-unanimity term of finite arity. Clearly, if the arity of the near-unanimity term of $\mathbf{A}$ is known, then finding the near-unanimity term is easy by simply calculating the free algebra generated by the appropriate number of elements in the variety generated by $\mathbf{A}$. The difficulty lies in the fact that we do not have an upper bound for the arity of a possible near-unanimity term.

The near-unanimity problem was posed in [3] over ten years ago, and motivated by the natural duality problem: given a finite algebra, decide if the quasi-variety it generates admits a natural duality (see [2] for details). B. A. Davey and H. Werner proved in [4] that in the presence of a near-unanimity term of $\mathbf{A}$, the quasi-variety $\mathcal{Q}$ generated by $\mathbf{A}$ admits a natural duality. The converse was proved by B. A. Davey, L. Heindorf and R. McKenzie in [3] under the assumption that $\mathcal{Q}$ is congruence join-semi-distributive: if $\mathcal{Q}$ admits a natural duality and is congruence join-semidistributive then $\mathbf{A}$ has a (finitary) near-unanimity term. This theorem, known as the near-unanimity obstacle theorem, implies that if the near-unanimity problem were undecidable, then the natural duality problem would also be undecidable, but not conversely.

Clearly, an algebra $\mathbf{A}$ has a near-unanimity term operation $t$ if and only if the equations

$$
t(y, x, \ldots, x)=t(x, y, x, \ldots, x)=\cdots=t(x, \ldots, x, y)=x
$$

hold for the generator elements $x, y$ of the two-generated free algebra in the variety generated by A. Probably this observation motivated R. McKenzie's unpublished result [7] where he proves that it is undecidable of a finite algebra $\mathbf{A}$ and a pair $x, y \in A$ of fixed elements whether $\mathbf{A}$ has a term $t$ that behaves as a near-unanimity

While working on this paper, the author was partially supported by the Hungarian National Foundation for Scientific Research (OTKA) grant nos. T 37877 and T 60148. 
term on $\{x, y\}$. His result does not imply the undecidability of the near-unanimity problem because the algebras used in his construction are not freely generated by the elements $x, y$ in the variety they generate.

The key result presented in this paper is the improvement of R. McKenzie's result to a fixed $|A|-2$ element subset, and the simplification of his construction. The basic idea, however, is intact: the use of Minsky machines - which are equivalent to Turing machines - and the encoding of their computations in "slim" terms of $\mathbf{A}$. The method used in the proof relies on an absorbing element as the indicator of defects. The existence of this absorbing element prevents the further improvement of this approach to prove the undecidability of the near-unanimity problem. However, an improvement to $|A|-1$ elements might be possible, which could be formulated, analogously to the results in [5], as the undecidability of the near-unanimity problem for partial algebras:

Problem. Given a finite partial algebra, decide whether it has a term that is defined on all near-unanimous assignments and satisfies the near-unanimity identities.

It is natural to attack the near-unanimity problem from the other perspective, as well: try to prove that it is decidable. We have tried the divide-and-conquer approach using I. Rosenberg's characterization of maximal clones. It turns out that in three of the six classes of maximal clones the problem is decidable. If we restrict ourselves to idempotent algebras, then we can further eliminate one of the three remaining classes. In the idempotent case the best result is obtained using Á. Szendrei's characterization of idempotent strictly simple term minimal algebras [14].

The two parts of the paper, partial results on the undecidability and decidability of the near-unanimity problem, are not dependent on each other. We assume only basic knowledge of universal algebra and direct the reader to either [1] or [8] for reference.

The author is indebted to R. McKenzie, Á. Szendrei and J. Ježek for their invaluable comments and directions.

\section{UNDECIDABILITY OF A PARTIAL NU-TERM}

Definition 2.1. Let $\mathbf{A}$ be a fixed finite algebra, $t\left(x_{1}, \ldots, x_{n}\right)$ be a term of $\mathbf{A}$, and $S$ be a subset of $A$. We say that $t$ is a partial near-unanimity term on $S$ if $t\left(y, \ldots, y, x_{i}, y, \ldots, y\right)=y$ for all $1 \leq i \leq n$ and $x_{i}, y \in S$.

Note that a term $t$ of $\mathbf{A}$ is a NU-term iff it is a partial NU-term on $A$. Now one can ask the decidability of a partial NU-term on some subset. It was proved in [7] that the existence of a partial NU-term on a fixed two-element subset is undecidable. We will extend this result to a subset excluding two fixed elements, which is our main result in this section.

Theorem 2.2. There exists no algorithm that can decide of a finite algebra $\mathbf{A}$ and two fixed elements $r, w \in A$ if $\mathbf{A}$ has a near-unanimity term on the set $A \backslash\{r, w\}$.

Following the proof of R. McKenzie, our work is based on the undecidability of the halting problem for Minsky machines. The Minsky machine was invented by M. Minsky in 1961 (see $[9,10]$ ), but he writes that the concept was inspired by some ideas of M. O. Rabin and D. Scott [12]. The "hardware" of a Minsky machine $\mathcal{M}$ consists of two registers $A$ and $B$, which can contain arbitrary natural numbers. 
The "software" is a finite set $S$ of states together with a list of commands. There are two special states: the initial state $q_{1} \in S$, and the halting state $q_{0} \in S$. The machine starts in the initial state, stops at the halting state, and at any given time it is in one of the states. For each state $i \in S \backslash\left\{q_{0}\right\}$ there is a single command which describes the state-transition from state $i$ together with the change of the registers' contents. There are two types of commands:

- in state $i$ increase register $X$ by one and go to state $j$, and

- in state $i$ if register $X$ contains zero, then go to state $j$, otherwise decrease $X$ by one and go to state $k$.

Now we give the formal definition.

Definition 2.3. A Minsky machine $\mathcal{M}=\left\langle S, q_{0}, q_{1}, M\right\rangle$ is a finite set $S$ of states with two distinguished elements $q_{0}, q_{1} \in S$ together with a mapping

$$
M: S \backslash\left\{q_{0}\right\} \rightarrow\{\langle X, j\rangle,\langle X, j, k\rangle \mid X \in\{A, B\} \text { and } j, k \in S\} .
$$

We call $q_{0}$ the halting state, and $q_{1}$ the initial state. The symbols $A$ and $B$ represent the registers.

The mapping $M$ describes the commands of $\mathcal{M}$ in the following way. For any given state $i \in S \backslash\left\{q_{0}\right\}$ the tuple $M(i)$ is either of the form $\langle X, j\rangle$ or $\langle X, j, k\rangle$, which correspond to the two types of commands described earlier.

Definition 2.4. A configuration $\langle i, a, b\rangle$ of $\mathcal{M}$ is an element of $S \times \mathbb{N} \times \mathbb{N}$, which specifies the current state and the values of the registers. We call $\langle i, a, b\rangle$ an initial configuration (halting configuration) if $i=q_{1}$ (or $i=q_{0}$, respectively).

For any configuration the Minsky machine $\mathcal{M}$ uniquely determines (computes) the next configuration. By iteration, starting from the initial configuration with zero valued registers, we obtain a sequence of configurations, which will be called the computation of $\mathcal{M}$.

Definition 2.5. The processor for $\mathcal{M}$ is a partial mapping of the set of configurations into itself denoted by $\overline{\mathcal{M}}$ and defined as

$$
\overline{\mathcal{M}}(\langle i, a, b\rangle)= \begin{cases}\text { undefined } & \text { if } i=q_{0}, \\ \langle j, a+1, b\rangle & \text { if } M(i)=\langle A, j\rangle, \\ \langle j, 0, b\rangle & \text { if } M(i)=\langle A, j, k\rangle \text { and } a=0, \\ \langle k, a-1, b\rangle & \text { if } M(i)=\langle A, j, k\rangle \text { and } a>0, \\ \langle j, a, b+1\rangle & \text { if } M(i)=\langle B, j\rangle, \\ \langle j, a, 0\rangle & \text { if } M(i)=\langle B, j, k\rangle \text { and } b=0, \\ \langle k, a, b-1\rangle & \text { if } M(i)=\langle B, j, k\rangle \text { and } b>0 .\end{cases}
$$

We will use iterative applications of the processor $\overline{\mathcal{M}}$ and adopt the power notation defined as $\overline{\mathcal{M}}^{0}(\langle i, a, b\rangle)=\langle i, a, b\rangle$ and $\overline{\mathcal{M}}^{n+1}(\langle i, a, b\rangle)=\overline{\mathcal{M}}\left(\overline{\mathcal{M}}^{n}(\langle i, a, b\rangle)\right)$. Note that $\overline{\mathcal{M}}^{n}(\langle i, a, b\rangle)$ is undefined if and only if $\overline{\mathcal{M}}^{m}(\langle i, a, b\rangle)$ is a halting configuration for some $m<n$.

Definition 2.6. We say that $\mathcal{M}$ halts if it halts on the $\langle 0,0\rangle$ input, that is, if $\overline{\mathcal{M}}^{n}\left(\left\langle q_{1}, 0,0\right\rangle\right)$ is a halting configuration for some $n>0$. 
It is proved in [9] that Minsky machines are equivalent to Turing machines in the following sense. Given a Minsky machine $\mathcal{M}$ (or Turing machine $\mathcal{T}$ ), we can algorithmically construct a Turing machine $\mathcal{T}(\mathcal{M})$ (or Minsky machine $\mathcal{M}(\mathcal{T})$ ) which halts if and only if the original machine halts. This means that the halting problem for Minsky machines is as difficult as for Turing machines; that is, undecidable. Thus a new path opens for proving the undecidability of algebraic problems by interpreting Minsky machines. For example this route was taken in [6] to prove the undecidability of various kinds of word problems.

In the rest of this section we are going to prove Theorem 2.2 in the following way. For any Minsky machine $\mathcal{M}$ we define an algebra $\mathbf{A}(\mathcal{M})$ with two special elements $r, w \in A(\mathcal{M})$ such that $\mathbf{A}(\mathcal{M})$ will have a partial near-unanimity term on $A(\mathcal{M}) \backslash\{r, w\}$ if and only if $\mathcal{M}$ halts. This is clearly enough since the halting problem for Minsky machines is undecidable.

Let $S$ be the set of states of $\mathcal{M}$ with two special states: the initial state $q_{1} \in S$ and the halting state $q_{0} \in S$. Let the symbols $A$ and $B$ denote the registers of $\mathcal{M}$. For each $i \in S \backslash\left\{q_{0}\right\}$ there is a unique command which is either of the form

- $i$ : inc $R, j$ (increase register $R \in\{A, B\}$ by one and go to state $j \in S$ ), or

- $i$ : dec $R, j, k$ (if register $R \in\{A, B\}$ contains zero, then go to state $j \in S$, otherwise decrease register $R$ by one and go to state $k \in S$ ).

$\operatorname{By} \operatorname{maj}(x, y, z)$ we denote the majority element of $\{x, y, z\}$, i.e., the element that appears at least twice among $x, y$ and $z$ if such element exists, otherwise $\operatorname{maj}(x, y, z)$ is undefined. Formally,

$$
\operatorname{maj}(x, y, z)= \begin{cases}x & \text { if } x=y \text { or } x=z \\ y & \text { if } y=x \text { or } y=z \\ z & \text { if } z=x \text { or } z=y \\ \text { undef. } & \text { otherwise }\end{cases}
$$

Now we define the algebra $\mathbf{A}(\mathcal{M})$ in full detail.

Definition 2.7. Let $C=\{0, A, B, 1\}$. We define the algebra $\mathbf{A}(\mathcal{M})$ on the set $A(\mathcal{M})=S \times C \cup\{p, r, w\}$ with the following operations

$$
\begin{gathered}
I(x)= \begin{cases}w & \text { if } x \in\{r, w\} \\
\left\langle q_{1}, 0\right\rangle & \text { if } x=p \\
r & \text { if } x \in S \times C\end{cases} \\
M(x, y, z, u)=\left\{\begin{aligned}
\operatorname{maj}(y, z, u) & \text { if }\{y, z, u\} \cap\{w, r\}=\emptyset, \operatorname{maj}(y, z, u) \text { exists } \\
p & \text { and } \operatorname{maj}(y, z, u) \neq p,
\end{aligned}\right. \\
\begin{aligned}
& \operatorname{maj}\{y, z, u\} \cap\{w, r\}=\emptyset, \operatorname{maj}(y, z, u) \text { exists } \\
& w \text { otherwise; }
\end{aligned}
\end{gathered}
$$


for each state $i \in S \backslash\left\{q_{0}\right\}$ for which $M(i)=\langle R, j\rangle$ where $R \in\{A, B\}$ and $j \in S$, that is, for each command of $\mathcal{M}$ of the form $i:$ inc $R, j$, the operation

$$
F_{i}(x, y)= \begin{cases}\langle j, c\rangle & \text { if } x=\langle i, c\rangle \text { and } y=p, \\ \langle j, R\rangle & \text { if } x=\langle i, 0\rangle \text { and } y \in S \times C, \\ r & \text { if } x=r \text { and } y=p \\ w & \text { otherwise }\end{cases}
$$

for each state $i \in S \backslash\left\{q_{0}\right\}$ for which $M(i)=\langle R, j, k\rangle$ where $R \in\{A, B\}$ and $j, k \in S$, that is, for each command of $\mathcal{M}$ of the form $i: \operatorname{dec} R, j, k$, the operation

$$
\begin{aligned}
G_{i}(x, y) & = \begin{cases}\langle k, c\rangle & \text { if } x=\langle i, c\rangle \text { and } y=p, \\
\langle k, 1\rangle & \text { if } x=\langle i, R\rangle \text { and } y \in S \times C, \\
r & \text { if } x=r \text { and } y=p, \\
w & \text { otherwise; }\end{cases} \\
H_{i}(x) & = \begin{cases}\langle j, c\rangle & \text { if } x=\langle i, c\rangle \text { and } c \neq R, \\
r & \text { if } x=r, \\
w & \text { otherwise. }\end{cases}
\end{aligned}
$$

We will investigate this algebra in detail. The first important property of $\mathbf{A}(\mathcal{M})$ is that it almost has an absorbing element.

Definition 2.8. Let $A$ be a set, and $f: A^{n} \rightarrow A$. An element $w \in A$ is absorbing for $f$ if $f(\bar{a})=w$ whenever $\bar{a} \in A^{n}$ and $w \in\left\{a_{1}, \ldots, a_{n}\right\}$.

Lemma 2.9. The element $w$ of $\mathbf{A}(\mathcal{M})$ is absorbing for the operations $I, F_{i}, G_{i}$ and $H_{i}$.

Proof. One only has to check the definition of $\mathbf{A}(\mathcal{M})$. In the definition of $I$ this is stated explicitly. In the definition of $F_{i}, G_{i}$ and $H_{i}$ only the 'otherwise' case can be applied.

Note that $w$ is not an absorbing element for the operation $M$, but almost, except in the first variable. Combining this with the previous lemma one can see that $\mathbf{A}(\mathcal{M})$ cannot have a partial NU-term on a nontrivial subset that includes $w$. For example if the rightmost variable of a term is evaluated with $w$, then the term always yields $w$. We will use the element $w$ to indicate some irregularity of a term.

Definition 2.10. Let $\bar{x}=\left(x_{1}, x_{2}, \ldots\right)$ be a fixed countably infinite list of variables, and $\bar{p}$ be the constant $p$ assignment for these variables. For each element $e \in A(\mathcal{M})$ let $\left.\bar{p}\right|_{x_{n}=e}$ be the assignment $x_{n}=e$ and $x_{m}=p$ if $m \neq n$. We say that a term $t(\bar{x})$ is regular if $t(\bar{p}) \neq w$ and $t\left(\left.\bar{p}\right|_{x_{n}=e}\right) \neq w$ for each $n \in \mathbb{N}$ and $e \in S \times C$.

Definition 2.11. We define slim terms inductively. The term $I\left(x_{n}\right)$ is slim for every variable $x_{n}$. If $t$ is slim, then so are $F_{i}(t, y), G_{i}(t, y)$ and $H_{i}(t)$ for any state $i \in S$ and variable $y \in\left\{x_{1}, x_{2}, \ldots\right\}$.

Lemma 2.12. Every regular term $t$ in which the operation symbol $M$ does not appear is either slim or a variable. Moreover, if $t$ is regular and slim, then there exists an assignment $\left.\bar{p}\right|_{x_{n}=e}$ for some $x_{n}$ and $e \in S \times C$, such that $t\left(\left.\bar{p}\right|_{x_{n}=e}\right)=r$. 
Proof. Assume that $t$ is regular and that the operation symbol $M$ does not appear in $t$. We use induction on the complexity of $t$. Note that $t$ cannot be a variable because variables are not slim by definition, therefore the leftmost symbol of $t$ (in prefix notation) is either $I, F_{i}, G_{i}$ or $H_{i}$.

Suppose that $t(\bar{x})=I\left(t_{1}(\bar{x})\right)$. Because of Lemma 2.9 we know that $t_{1}$ must be regular, as well. If $t_{1}$ is not a variable, then according to our assumption we have an assignment $\left.\bar{p}\right|_{x_{n}=e}$ such that $t_{1}\left(\left.\bar{p}\right|_{x_{n}=e}\right)=r$. This shows that $t\left(\left.\bar{p}\right|_{x_{n}=e}\right)=I(r)=w$, which is a contradiction. Thus $t_{1}$ must be a variable, that is, $t=I\left(x_{n}\right)$ for some integer $n$. In this case, $t$ is clearly slim. Moreover, for any element $e \in S \times C$, $t\left(\left.\bar{p}\right|_{x_{n}=e}\right)=I(e)=r$ by definition.

Now suppose that $t(\bar{x})=F_{i}\left(t_{1}(\bar{x}), t_{2}(\bar{x})\right)$ for some $i \in S$. Again, both $t_{1}$ and $t_{2}$ must be regular. If $t_{1}$ is a variable, then $t(\bar{p})=F_{i}\left(p, t_{2}(\bar{p})\right)=w$. Thus $t_{1}$ cannot be a variable. So there exists an assignment $\left.\bar{p}\right|_{x_{n}=e}$ such that $t_{1}\left(\left.\bar{p}\right|_{x_{n}=e}\right)=r$, which forces $t_{2}\left(\left.\bar{p}\right|_{x_{n}=e}\right)=p$. But $p$ is not in the range of any of the operations $I, F_{i}, G_{i}$ and $H_{i}$; thus $t_{2}$ must be a variable. This proves that $t$ is slim and that $t\left(\left.\bar{p}\right|_{x_{n}=e}\right)=F_{i}(r, p)=r$.

The same argument works if the leftmost operation symbol of $t$ is either $G_{i}$ or $H_{i}$.

Regular slim terms play a very important role in the proof; they essentially encode the computation of the Minsky machine $\mathcal{M}$. To see how this works, we describe the construction of a partial near-unanimity term from a halting computation.

Lemma 2.13. If $\mathcal{M}$ halts, then there exists a partial near-unanimity term on $A(\mathcal{M}) \backslash\{r, w\}$.

Proof. We use the processor $\overline{\mathcal{M}}^{n}$ from Definition 2.5. Assume that $\mathcal{M}$ halts in $n$ steps, that is, $\overline{\mathcal{M}}^{n}\left(\left\langle q_{1}, 0,0\right\rangle\right)=\left\langle q_{0},-,-\right\rangle$. For each natural number $m \leq n$ we define $i_{m}, a_{m}$ and $b_{m}$ by

$$
\overline{\mathcal{M}}^{m}\left(\left\langle q_{1}, 0,0\right\rangle\right)=\left\langle i_{m}, a_{m}, b_{m}\right\rangle .
$$

We are going to build a slim term of depth $n+1$ by induction. Put $t_{0}=I(x)$. Now suppose that $t_{m}$ is already defined. At step $m$ the machine is in state $i_{m}$. There is a unique command for each state.

If the command for state $i_{m}$ is of the form $i$ : inc $R, j$, then put $t_{m+1}=$ $F_{i_{m}}\left(t_{m}, y_{m}\right)$ where $y_{m}$ is a new variable. Now assume that the command for state $i_{m}$ is of the form $i: \operatorname{dec} R, j, k$ where $R=A$. If $a_{m}=0$, then put $t_{m+1}=H_{i_{m}}\left(t_{m}\right)$. If $a_{m} \neq 0$, then let $m^{\prime}<m$ be the largest natural number such that $a_{m^{\prime}}<a_{m}$, and put $t_{m+1}=G_{i_{m}}\left(t_{m}, y_{m^{\prime}}\right)$. The case when $R=B$ is handled similarly using $b_{m}$ and $b_{m^{\prime}}$ instead of $a_{m}$ and $a_{m^{\prime}}$.

Finally, put $t=M\left(t_{n}, z_{1}, z_{2}, z_{3}\right)$ where $z_{1}, z_{2}$ and $z_{3}$ are new variables. We claim that $t_{n}$ is a regular slim term and $t$ is a near-unanimity term on $A(\mathcal{M}) \backslash\{r, w\}$.

Claim 1. The term $t_{n}$ is slim.

This follows from the construction. We have used only variables in the second coordinates of $F_{i}$ and $G_{i}$.

Claim 2. No variable of thas more than two occurrences. If a variable has exactly two occurrences, then it is $y_{m^{\prime}}$ for some $m$ and the two occurrences are at $t_{m^{\prime}+1}=F_{i_{m^{\prime}}}\left(t_{m^{\prime}}, y_{m^{\prime}}\right)$ and $t_{m+1}=G_{i_{m}}\left(t_{m}, y_{m^{\prime}}\right)$. If a variable $y_{m}$ has exactly one occurrence, then it is at $t_{m+1}=F_{i_{m}}\left(t_{m}, y_{m}\right)$. 
The variables $x, z_{1}, z_{2}$ and $z_{3}$ have single occurrences. At each $F_{i}$ we always introduced a new variable. Now consider the case when $t_{m+1}=G_{i_{m}}\left(t_{m}, y_{m^{\prime}}\right)$. From the definition we know that $a_{m^{\prime}}<a_{m}$ and $a_{m} \leq a_{m^{\prime}+1}, \ldots, a_{m}$ (assuming that $R=A$ ). Since $a_{m^{\prime}}<a_{m} \leq a_{m^{\prime}+1}$ and the machine cannot increase a register by more than one, $a_{m^{\prime}}+1=a_{m}=a_{m^{\prime}+1}$. This implies that the command for state $i_{m^{\prime}}$ is of the form $i$ : inc $R, j$ and $R=A$. On the other hand, the command for state $i_{m}$ is of the form $i: \operatorname{dec} A, j, k$ and $a_{m} \neq 0$, therefore $a_{m+1}=a_{m}-1$. To summarize, for each pair $\left\langle m^{\prime}, m\right\rangle$

$$
\begin{gathered}
a_{m^{\prime}}+1=a_{m^{\prime}+1}=a_{m}=a_{m+1}+1, \text { and } \\
a_{m} \leq a_{m^{\prime}+1}, \ldots, a_{m} .
\end{gathered}
$$

Note that this condition is symmetric. If $m^{\prime}$ is in pair with some $m$, then $m$ is the least natural number such that $m^{\prime}<m$ and $a_{m^{\prime}+1}>a_{m+1}$. Therefore, $y_{m^{\prime}}$ has at most two occurrences.

Claim 3. $t_{m}(\bar{p})=\left\langle i_{m}, 0\right\rangle$ for all $m \leq n$

We prove by induction on $m$. For $m=0$ this is true by definition: $I(p)=$ $\left\langle q_{1}, 0\right\rangle$. Now we prove it for $m+1$. By definition $t_{m+1}$ is $F_{i_{m}}\left(t_{m}, y_{m}\right), H_{i_{m}}\left(t_{m}\right)$ or $G_{i_{m}}\left(t_{m}, y_{m^{\prime}}\right)$. Therefore $t_{m+1}(\bar{p})$ is $F_{i_{m}}\left(\left\langle i_{m}, 0\right\rangle, p\right), H_{i_{m}}\left(\left\langle i_{m}, 0\right\rangle\right)$ or $G_{i_{m}}\left(\left\langle i_{m}, 0\right\rangle, p\right)$.

If $t_{m+1}(\bar{p})=F_{i_{m}}\left(\left\langle i_{m}, 0\right\rangle, p\right)$, then the command for state $i_{m}$ is of the form $i$ : inc $R, j$. Thus $F_{i_{m}}\left(\left\langle i_{m}, 0\right\rangle, p\right)=\langle j, 0\rangle$ by Definition 2.7, and $\mathcal{M}\left(\left\langle i_{m}, a_{m}, b_{m}\right\rangle\right)=$ $\langle j,-,-\rangle$ by Definition 2.5. Therefore $j=i_{m+1}$ and consequently $t_{m+1}(\bar{p})=$ $\left\langle i_{m+1}, 0\right\rangle$.

If $t_{m+1}(\bar{p})=H_{i_{m}}\left(\left\langle i_{m}, 0\right\rangle\right)$, then the command for state $i_{m}$ is of the form $i$ : $\operatorname{dec} R, j, k$, moreover $a_{m}=0$ if $R=A$, and $b_{m}=0$ if $R=B$. Now $H_{i_{m}}\left(\left\langle i_{m}, 0\right\rangle, p\right)=$ $\langle j, 0\rangle$ by Definition 2.7, and $\mathcal{M}\left(\left\langle i_{m}, a_{m}, b_{m}\right\rangle\right)=\langle j,-,-\rangle$ by Definition 2.5. Therefore $j=i_{m+1}$ and consequently $t_{m+1}(\bar{p})=\left\langle i_{m+1}, 0\right\rangle$.

Finally, if $t_{m+1}(\bar{p})=G_{i_{m}}\left(\left\langle i_{m}, 0\right\rangle, p\right)$, then the command for state $i_{m}$ is of the form $i$ : $\operatorname{dec} R, j, k$, moreover $a_{m}>0$ if $R=A$, and $b_{m}>0$ if $R=B$. Now $G_{i_{m}}\left(\left\langle i_{m}, 0\right\rangle, p\right)=\langle k, 0\rangle$ by Definition 2.7 , and $\mathcal{M}\left(\left\langle i_{m}, a_{m}, b_{m}\right\rangle\right)=\langle k,-,-\rangle$ by Definition 2.5. Therefore $k=i_{m+1}$ and consequently $t_{m+1}(\bar{p})=\left\langle i_{m+1}, 0\right\rangle$.

Claim 4. $t_{m}\left(\left.\bar{p}\right|_{x=e}\right)=r$ for all $m \leq n$ and $e \in S \times C$ where $x$ is the variable used to define $t_{0}=I(x)$.

We prove the claim by induction. For $m=0, t_{0}=I(x)$ and $t_{0}\left(\left.\bar{p}\right|_{x=e}\right)=I(e)=r$. Now assume that $t_{m}\left(\left.\bar{p}\right|_{x=e}\right)=r$ for some $m<n$. By the construction, $t_{m+1}$ is either $F_{i_{m}}\left(t_{m}, y\right), G_{i_{m}}\left(t_{m}, y\right)$ or $H_{i_{m}}\left(t_{m}\right)$ where $y$ is some variable different from $x$. Thus $t_{m+1}\left(\left.\bar{p}\right|_{x=e}\right)$ equals either $F_{i_{m}}(r, p), G_{i_{m}}(r, p)$ or $H_{i_{m}}(r)$. But each of these equals $r$ by Definition 2.7 .

Claim 5. Let $h<n$ and $e \in S \times C$ be fixed and assume that $y_{h}$ has exactly one occurrence in $t_{n}$. Let $R$ be the register manipulated in the command for state $i_{h}$. Then

$$
t_{m}\left(\left.\bar{p}\right|_{y_{h}=e}\right)= \begin{cases}\left\langle i_{m}, 0\right\rangle & \text { if } 0 \leq m \leq h, \\ \left\langle i_{m}, R\right\rangle & \text { if } h<m \leq n .\end{cases}
$$

Without loss of generality we can assume that $R=A$. By Claim 2, the single occurrence of $y_{h}$ is at $t_{h+1}=F_{i_{h}}\left(t_{h}, y_{h}\right)$. Therefore, if $m \leq h$, then $t_{m}\left(\left.\bar{p}\right|_{y_{h}=e}\right)=$ $t_{m}(\bar{p})=\left\langle i_{m}, 0\right\rangle$ by Claim 3 . We use induction on $m$ to prove the other case. For the base of the induction we have $t_{h+1}\left(\left.\bar{p}\right|_{y_{h}=e}\right)=F_{i_{h}}\left(\left\langle i_{h}, 0\right\rangle, e\right)=\left\langle i_{h+1}, A\right\rangle$. 
Now consider the induction step from $m$ to $m+1$. Assume that $t_{m+1}=$ $F_{i_{m}}\left(t_{m}, y_{m}\right)$. Since $y_{h}$ has a single occurrence, $y_{h} \neq y_{m}$, and thus $t_{m+1}\left(\left.\bar{p}\right|_{y_{h}=e}\right)=$ $F_{i_{m}}\left(\left\langle i_{m}, A\right\rangle, p\right)=\left\langle i_{m+1}, A\right\rangle$. The same argument works when $t_{m+1}=G_{i_{m}}\left(t_{m}, y_{m^{\prime}}\right)$.

Now assume that $t_{m+1}=H_{i_{m}}\left(t_{m}\right)$. From equation $(*)$ in the proof of Claim 2 we can see that $a_{h}<a_{h+1}, \ldots, a_{n}$, otherwise $y_{h}$ would have at least two occurrences. Therefore, $a_{m} \neq 0$. By the definition of $t_{m+1}$ we know that either $a_{m}$ or $b_{m}$ must be zero. Thus it is register $B$ which is manipulated in the command for state $i_{m}$. This implies that $t_{m+1}\left(\left.\bar{p}\right|_{y_{h}=e}\right)=H_{i_{m}}\left(\left\langle i_{m}, A\right\rangle\right)=\left\langle i_{m+1}, A\right\rangle$.

Claim 6. Let $h<n$ and $e \in S \times C$ be fixed and assume that $y_{h^{\prime}}$ has exactly two occurrences in $t_{n}$ as described in Claim 2. Let $R$ be the register manipulated in the commands for states $i_{h^{\prime}}$ and $i_{h}$. Then

$$
t_{m}\left(\left.\bar{p}\right|_{y_{h^{\prime}}=e}\right)= \begin{cases}\left\langle i_{m}, 0\right\rangle & \text { if } 0 \leq m \leq h^{\prime}, \\ \left\langle i_{m}, R\right\rangle & \text { if } h^{\prime}<m \leq h, \\ \left\langle i_{m}, 1\right\rangle & \text { if } h<m \leq n .\end{cases}
$$

Without loss of generality we can assume that $R=A$. For the first two cases of the displayed equation above the same argument works as in the previous claim, but using $h^{\prime}$ instead of $h$.

We prove the third case by induction on $m$. For the base of the induction we have $t_{h+1}=G_{i_{h}}\left(t_{h}, y_{h^{\prime}}\right)$. Hence $t_{h+1}\left(\left.\bar{p}\right|_{y_{h^{\prime}}=e}\right)=G_{i_{h}}\left(\left\langle i_{h}, A\right\rangle, e\right)=\left\langle i_{h+1}, 1\right\rangle$. The induction step is now easy as there are no other occurrences of $y_{h^{\prime}}$ along the term $t_{n}$. Therefore, we always calculate $F_{i_{m}}\left(\left\langle i_{m}, 1\right\rangle, p\right), G_{i_{m}}\left(\left\langle i_{m}, 1\right\rangle, p\right)$, or $H_{i_{m}}\left(\left\langle i_{m}, 1\right\rangle\right)$, which all yield $\left\langle i_{m+1}, 1\right\rangle$.

Claim 7. The term $t_{n}$ is regular. Moreover, $t_{n}\left(\left.\bar{p}\right|_{u=e}\right) \in\left\{q_{0}\right\} \times C \cup\{r\}$ for all variables $u$ and all $e \in A(\mathcal{M}) \backslash\{r, w\}$.

Take any element $e \in S \times C$. By Claims 3 and 4 we have $t_{n}(\bar{p})=\left\langle q_{0}, 0\right\rangle$ and $t_{n}\left(\left.\bar{p}\right|_{x=e}\right)=r$, respectively. Now take a variable $y_{h}$. If $y_{h}$ has no occurrence in $t_{n}$, then $t_{n}\left(\left.\bar{p}\right|_{y_{h}=e}\right)=t_{n}(\bar{p})=\left\langle q_{0}, 0\right\rangle$. Otherwise $y_{h}$ has one or two occurrences by Claim 2. Then by Claims 5 and 6 we have $t_{n}\left(\left.\bar{p}\right|_{y=e}\right) \in\left\{q_{0}\right\} \times C$.

ClaIm 8. $t$ is a near-unanimity term on $A(\mathcal{M}) \backslash\{r, w\}$.

Take a near-unanimous assignment $\bar{a}$ on $A(\mathcal{M}) \backslash\{r, w\}$. If the majority element is not $p$, then $t(\bar{a})=M\left(t_{n}(\bar{a}), z_{1}, z_{2}, z_{3}\right)=\operatorname{maj}\left(z_{1}, z_{2}, z_{3}\right)$. If the majority element is $p$, then $t_{n}(\bar{a}) \in\left\{q_{0}\right\} \times C \cup\{r\}$ by Claim 7 , and hence $t(\bar{a})=p$. Therefore, $t$ is a near-unanimity term on $A(\mathcal{M}) \backslash\{r, w\}$.

We have seen how to encode the halting computation into the regular slim term $t_{n}$. Our goal now is the reverse; to show that the computation of $\mathcal{M}$ can be recovered from a regular slim term.

Lemma 2.14. Let $t_{n}$ be a regular slim term of depth $n+1$. Then $t_{n}(\bar{p})=\left\langle i_{n}, 0\right\rangle$ where $i_{n}$ is the state of the machine $\mathcal{M}$ after the first $n$ steps.

Proof. We want to show that the term $t_{n}$ behaves the same way as the one in the proof of the previous lemma. Denote by $t_{m}$ the unique subterm of $t_{n}$ of depth $m+1$. That is, $t_{0}=I(-)$, and $t_{m+1}$ is $F_{i}\left(t_{m},-\right), G_{i}\left(t_{m},-\right)$ or $H_{i}\left(t_{m}\right)$ for some $i \in S$.

Claim 1. $t_{m}\left(\left.\bar{p}\right|_{x=e}\right) \neq w$ for all $m \leq n, e \in S \times C$ and all variables $x$ of $t_{n}$. 
Suppose that $t_{m}\left(\left.\bar{p}\right|_{x=e}\right)=w$ for some $m \leq n, e \in S \times C$ and variable $x$ of $t_{n}$. The element $w$ is absorbing for the operations of $t_{n}$ by Lemma 2.9, which implies that $t_{n}\left(\left.\bar{p}\right|_{x=e}\right)=w$. This contradicts the regularity of $t_{n}$ and proves the claim.

Claim 2. $t_{m}(\bar{p}) \in S \times\{0\}$ for all $m \leq n$.

We prove the claim by induction on $m$. For $m=0$ we have $t_{0}(\bar{p})=I(p)=$ $\left\langle q_{1}, 0\right\rangle \in S \times\{0\}$. For the induction step, assume that $t_{m}(\bar{p})=\langle s, 0\rangle \in S \times\{0\}$ and consider $t_{m+1}$. If $t_{m+1}=F_{i}\left(t_{m}, y\right)$ for some $i \in S$ and variable $y$, then $t_{m+1}(\bar{p})=$ $F_{i}(\langle s, 0\rangle, p)$ which equals either $\langle j, 0\rangle$ for some $j \in S$ or $w$. But $t_{m+1}(\bar{p}) \neq \omega$ as $t_{m+1}$ is regular, so $t_{m+1}(\bar{p})=\langle j, 0\rangle \in S \times\{0\}$. A similar argument works for the other two types of operations: $G_{i}$ and $H_{i}$.

Claim 3. Let $x$ be the variable used in $t_{0}$. Then $x$ has no other occurrence in $t_{n}$. Moreover, $t_{m}\left(\left.\bar{p}\right|_{x=e}\right)=r$ for all $m \leq n$ and $e \in S \times C$.

We use induction on $m$. For $m=0$ we have $t_{0}\left(\left.\bar{p}\right|_{x=e}\right)=I(e)=r$. For the induction step from $m$ to $m+1$ assume that $t_{m}\left(\left.\bar{p}\right|_{x=e}\right)=r$. Thus $t_{m+1}\left(\left.\bar{p}\right|_{x=e}\right)$ is $F_{i}(r, y), G_{i}(r, y)$ or $H_{i}(r)$ for some $i \in S$ and some variable $y$. We know that this value is not $w$ by Claim 1 . Looking up the definition of $F_{i}, G_{i}$ and $H_{i}$, we can see that the only choice is when the result is $r$ (and $y=p$ for $F_{i}$ and $G_{i}$ ). This completes the induction step and proves that $x \neq y($ as $e \neq p)$ when the operation is $F_{i}$ or $G_{i}$.

Claim 4. Assume that a variable $y \neq x$ has exactly one occurrence in $t_{n}$. Then the occurrence is at $t_{m+1}=F_{i}\left(t_{m}, y\right)$ for some $m<n$ and $i \in S$. Moreover, there exists no $h>m$ such that $t_{h+1}=H_{j}\left(t_{h}\right)$ and the command for $j$ manipulates the same register as the one for $i$.

Let $m$ be the least natural number such that $t_{m+1}$ has an occurrence of $y$. Then $t_{m+1}=F_{i}\left(t_{m}, y\right)$ or $t_{m+1}=G_{i}\left(t_{m}, y\right)$ for some $i \in S$. Take an element $e \in S \times C$, and consider $t_{m+1}\left(\left.\bar{p}\right|_{y=e}\right)$. By the choice of $m, t_{m}\left(\left.\bar{p}\right|_{y=e}\right)=t_{m}(\bar{p})$, and then by Claim $2, t_{m}\left(\left.\bar{p}\right|_{y=e}\right) \in S \times\{0\}$. Checking the definition of $G_{i}$ we see that $G_{i}\left(t_{m}\left(\left.\bar{p}\right|_{y=e}\right), e\right)=w$, a contradiction. So $t_{m+1}=F_{i}\left(t_{m}, y\right)$. Moreover, $t_{m+1}\left(\left.\bar{p}\right|_{y=e}\right) \in S \times\{R\}$ where $R$ is the register manipulated by the command for $i$. Now we show that $t_{h}\left(\left.\bar{p}\right|_{y=e}\right) \in S \times\{R\}$ for all $h>m$ by induction. For $m+1$ we already have this. For the induction step consider $a=t_{h+1}\left(\left.\bar{p}\right|_{y=e}\right)$. By definition $a$ is $F_{j}(\langle-, R\rangle, p), G_{j}(\langle-, R\rangle, p)$ or $H_{j}(\langle-, R\rangle)$ for some $j \in S$ and $a \neq w$. In the first two cases this shows that $a \in S \times\{R\}$. On the other hand, when $a=H_{j}(\langle-, R\rangle) \neq w$, then the command for state $j$ cannot manipulate the register $R$. This concludes the proof of this claim.

Claim 5. Assume that a variable $y \neq x$ has at least two occurrences in $t_{n}$. Then there exist $m^{\prime}<m$ such that $t_{m^{\prime}+1}=F_{i}\left(t_{m^{\prime}}, y\right), t_{m+1}=G_{j}\left(t_{m}, y\right)$ for some $i, j \in S$, the commands for $i$ and $j$ manipulate the same register $R$, and $y$ has no other occurrences than these two. Moreover, there exists no $m^{\prime}<h<m$ such that $t_{h+1}=H_{k}\left(t_{h}\right)$ and the command for $k$ manipulates the register $R$.

Let $m^{\prime}$ and $m$ be the least natural numbers such that $t_{m^{\prime}+1}$ has exactly one and $t_{m+1}$ has exactly two occurrences of $y$. The term $t_{m}$ has exactly one occurrence of $y$, so we can apply the previous claim. This proves half of the claim. It remains to be shown that $t_{m+1}=G_{j}\left(t_{m}, y\right)$ for some $j \in S$, that the command for $j$ manipulates the register $R$, and that there are no other occurrences of $y$. 
Fix an element $e \in S \times C$. From the proof of the previous claim we know that $t_{m}\left(\left.\bar{p}\right|_{y=e}\right) \in S \times\{R\}$ where $R$ is the register manipulated by the command for state $i$. Consider $a=t_{m+1}\left(\left.\bar{p}\right|_{y=e}\right)$. This element is either $F_{j}(\langle-, R\rangle, e)$ or $G_{j}(\langle-, R\rangle, e)$ for some $j$. Since $a \neq w$ by Claim (1), we must have $t_{m+1}=G_{j}\left(t_{m}, y\right)$, and the command for state $j$ must manipulate register $R$. Therefore, we have $t_{m+1}\left(\left.\bar{p}\right|_{y=e}\right) \in S \times\{1\}$.

Finally, we show that $t_{h}\left(\left.\bar{p}\right|_{y=e}\right) \in S \times\{1\}$ for all $h>m$ by induction. We have already the basis of the induction. To show the induction step, consider $t_{h+1}$. If $t_{h+1}=H_{k}\left(t_{h}\right)$ for some $k$, then we get $t_{h+1}\left(\left.\bar{p}\right|_{y=e}\right) \in S \times\{1\}$ by the definition of $H_{k}$. Now assume that $t_{h+1}=F_{k}\left(t_{h}, z\right)$. Since $t_{h+1}\left(\left.\bar{p}\right|_{y=e}\right) \neq w$ we must have $z \neq y$ and $t_{h+1}\left(\left.\bar{p}\right|_{y=e}\right) \in S \times\{1\}$. The same argument works for $G_{k}$, as well.

Claim 6. Let $i_{m}, a_{m}$ and $b_{m}$ be defined by $\overline{\mathcal{M}}^{m}\left(\left\langle q_{1}, 0,0\right\rangle\right)=\left\langle i_{m}, a_{m}, b_{m}\right\rangle$. Then the following hold for all $0 \leq m<n$.

(1) If the command for $i_{m}$ is of the form $i$ : inc $R, j$, then $t_{m+1}=F_{i_{m}}\left(t_{m},-\right)$.

(2) If the command for $i_{m}$ is of the form $i: \operatorname{dec} R, j, k$, and if $a_{m} \neq 0$ for $R=A$ while $b_{m} \neq 0$ for $R=B$, then $t_{m+1}=G_{i_{m}}\left(t_{m},-\right)$.

(3) If the command for $i_{m}$ is of the form $i: \operatorname{dec} R, j, k$, and if $a_{m}=0$ for $R=A$ while $b_{m}=0$ for $R=B$, then $t_{m+1}=H_{i_{m}}\left(t_{m},-\right)$.

Moreover, $t_{m}(\bar{p})=\left\langle i_{m}, 0\right\rangle$ for all $0 \leq m \leq n$.

We prove this by induction on $m$. For $m=0$ we have $t_{0}(\bar{p})=I(p)=\left\langle q_{1}, 0\right\rangle=$ $\left\langle i_{0}, 0\right\rangle$. For the induction step assume that $(1)-(3)$ hold for all $m^{\prime}<m$, a condition which is void if $m=0$, and $t_{m}(\bar{p})=\left\langle i_{m}, 0\right\rangle$. We have to show that $(1)-(3)$ hold for $m$ and $t_{m+1}(\bar{p})=\left\langle i_{m+1}, 0\right\rangle$.

Assume that $t_{m+1}=F_{i}\left(t_{m}, y\right)$ for some $i \in S$ and some variable $y$. We have to show that $i=i_{m}$ and $t_{m+1}(\bar{p})=\left\langle i_{m+1}, 0\right\rangle$. Since the operation $F_{i}$ is defined, the command for state $i$ is $i$ : inc $R, j$ for some $R \in\{A, B\}$ and $j \in S$. From the induction hypothesis, $t_{m}(\bar{p})=\left\langle i_{m}, 0\right\rangle$. Consider the element $e=t_{m+1}(\bar{p})=$ $F_{i}\left(\left\langle i_{m}, 0\right\rangle, p\right)$. Since $e \neq w$ by Claim (1), we must have $i=i_{m}$ and $e=\langle j, 0\rangle$. As $i_{m}=i$ and the command is $i$ : inc $R, j$, we have $i_{m+1}=j$. Therefore, $t_{m+1}(\bar{p})=$ $\left\langle i_{m+1}, 0\right\rangle$.

Assume that $t_{m+1}=G_{i}\left(t_{m}, y\right)$ for some $i \in S$ and variable $y$. We have to show that $i=i_{m}$ and $t_{m+1}(\bar{p})=\left\langle i_{m+1}, 0\right\rangle$. Since the operation $G_{i}$ is defined, the command for state $i$ is $i$ : $\operatorname{dec} R, j, k$ for some $R \in\{A, B\}$ and $j, k \in S$. Without loss of generality we can assume that $R=A$. Consider $e=t_{m+1}(\bar{p})=G_{i}\left(\left\langle i_{m}, 0\right\rangle, p\right)$. Since $e \neq w$, we must have $i=i_{m}$ and $e=\langle k, 0\rangle$. What remains to be shown is that $i_{m+1}=k$. We know that $i_{m+1}$ is either $j$ or $k$ depending on whether $a_{m}=0$ or $a_{m} \neq 0$. We claim that $a_{m} \neq 0$. By the definition of the Minsky machine, we have

$$
\begin{aligned}
a_{m} & =\mid\{h<m: \mathcal{M} \text { has increased register } A \text { at step } h\} \mid \\
& -\mid\{h<m: \mathcal{M} \text { has decreased register } A \text { at step } h\} \mid .
\end{aligned}
$$

Now using the induction hypothesis we get that

$$
\begin{aligned}
a_{m}=\mid\left\{h<m: t_{h+1}=F_{i_{h}}\left(t_{h},-\right)\right. \\
\left.\quad \text { and the command for } i_{h} \text { manipulates register } A\right\} \mid \\
-\mid\left\{h<m: t_{h+1}=G_{i_{h}}\left(t_{h},-\right)\right. \\
\left.\quad \text { and the command for } i_{h} \text { manipulates register } A\right\} \mid .
\end{aligned}
$$


Take a number $h$ from the second set $\mathrm{S}^{-}$, so $t_{h+1}=G_{i_{h}}\left(t_{h}, z\right)$ for some variable $z$, and the command for $i_{h}$ manipulates register $A$. By Claims 3,4 and 5 , the variable $z$ has exactly two occurrences; the other being at $t_{h^{\prime}+1}=F_{i_{h^{\prime}}}\left(t_{h}^{\prime}, z\right)$ for some $h^{\prime}<h$. Moreover, the command for $i_{h^{\prime}}$ manipulates the same register $A$. Thus $h^{\prime}$ belongs to the first set $\mathrm{S}^{+}$. This only shows that $a_{m} \geq 0$. But the same argument works for $t_{m+1}=G_{i}\left(t_{m}, y\right)$, showing that there exists an $m^{\prime}<m$ which belongs to $\mathrm{S}^{+}$, while $m \notin \mathrm{S}^{-}$. Therefore, $a_{m}>0$ and $i_{m+1}=k$.

Finally, assume that $t_{m+1}=H_{i}\left(t_{m}\right)$ for some $i \in S$. We have to show that $i=i_{m}$ and $t_{m+1}(\bar{p})=\left\langle i_{m+1}, 0\right\rangle$. Since the operation $H_{i}$ is defined, the command for state $i$ is $i$ : $\operatorname{dec} R, j, k$ for some $R \in\{A, B\}$ and $j, k \in S$. Without loss of generality we can assume that $R=A$. Consider $e=t_{m+1}(\bar{p})=H_{i}\left(\left\langle i_{m}, 0\right\rangle\right)$. Since $e \neq w$, we must have $i=i_{m}$ and $e=\langle j, 0\rangle$. What remains to be shown is that $i_{m+1}=j$. We know that $i_{m+1}$ is either $j$ or $k$ depending on whether $a_{m}=0$ or $a_{m} \neq 0$. To get a contradiction, suppose that $a_{m} \neq 0$, i.e., the set $\mathrm{S}^{+}$, defined in the previous subsection, has more elements than $\mathrm{S}^{-}$. We know that each element of $\mathrm{S}^{-}$is in pair with a unique element of $\mathrm{S}^{+}$. So there exists an $h<m$ such that $t_{h+1}=F_{i}\left(t_{h}, z\right)$ for some variable $z$, the command for $i$ manipulates register $A$, and $h$ is not in $\mathrm{S}^{-}$. Therefore, $z$ has exactly one occurrence in $t_{m}$. If $z$ has two occurrences, then the other one must appear after $t_{m+1}$. In any case, either by Claim 4 or 5 , the command for $i$ at $t_{m+1}=H_{i}\left(t_{m}\right)$ cannot manipulate register $A$. But according to our assumption it does, which is a contradiction. This shows that $a_{m}=0$, therefore $i_{m+1}=j$.

This finishes the proof of the last claim, which includes the statement $t_{n}(\bar{p})=$ $\left\langle i_{n}, 0\right\rangle$ of the lemma.

The previous two lemmas give the connection between regular slim terms and halting computations. What remains to be shown is that a regular slim term can be found as a subterm of a near-unanimity term on $A(\mathcal{M}) \backslash\{r, w\}$, or at least as a subterm of a "minimal" near-unanimity term.

Definition 2.15. Two terms $t_{1}$ and $t_{2}$ are $p$-equivalent iff $t_{1}(\bar{p})=t_{2}(\bar{p})$ and $t_{1}\left(\left.\bar{p}\right|_{x_{n}=e}\right)=t_{2}\left(\left.\bar{p}\right|_{x_{n}=e}\right)$ for each $n \in \mathbb{N}$ and $e \in S \times C$. A term is $p$-minimal iff there is no $p$-equivalent term of smaller complexity.

Lemma 2.16. Let $t$ be a regular p-minimal term in which the operation symbol $M$ appears. Then $\mathbf{A}(\mathcal{M})$ halts.

Proof. We use induction on the complexity of $t$. If $t=F_{i}\left(t_{1}, t_{2}\right)$, then both $t_{1}$ and $t_{2}$ must be regular (and $p$-minimal) by Lemma 2.9. So at least in one of them the operation symbol $M$ appears, and by induction we are done. The same argument works for the operations $G_{i}, H_{i}$ and $I$, as well.

Now suppose that $t=M\left(t_{1}, t_{2}, t_{3}, t_{4}\right)$. If $t_{2}, t_{3}$ or $t_{4}$ is not regular, then we have some near $p$-unanimous assignment $\bar{f}$ such that $w \in\left\{t_{2}(\bar{f}), t_{3}(\bar{f}), t_{4}(\bar{f})\right\}$. This forces $t(\bar{f})=w$, which is a contradiction. So $t_{2}, t_{3}$ and $t_{4}$ are regular. If in one of them the operation symbol $M$ appears, then we use induction on that subterm. So assume that $M$ does not occur in $t_{2}, t_{3}$ and $t_{4}$. By Lemma 2.12, each of them is either a slim term or a variable. If $t_{k}$ is $\operatorname{sim}(k \in\{2,3,4\})$, then we have an assignment $\left.\bar{p}\right|_{x_{n}=e}$ such that $t_{k}\left(\left.\bar{p}\right|_{x_{n}=e}\right)=r$. This forces a contradiction $t\left(\left.\bar{p}\right|_{x_{n}=e}\right)=w$. Thus $t_{2}, t_{3}$ and $t_{4}$ must be variables. If two of them are the same variable $y$, then $t$ is $p$-equivalent to $y$ because the operation $M$ must yield the majority element $y$, as it cannot return $w$ because $t$ is regular. This contradicts 
the $p$-minimality, thus the terms $t_{2}, t_{3}$ and $t_{4}$ are distinct variables. If $t_{1}$ is not regular, then we have an assignment $\left.\bar{p}\right|_{x_{n}=e}$ such that $t_{1}\left(\left.\bar{p}\right|_{x_{n}=e}\right)=w$. But this forces $t\left(\left.\bar{p}\right|_{x_{n}=e}\right)=w$, a contradiction. So $t_{1}$ must be regular. If $t_{1}$ contains the operation symbol $M$, then we use the induction. If $t_{1}$ does not contain $M$, then by Lemma 2.12 it is either a slim term or a variable. It cannot be a variable because $t(\bar{p}) \neq w$. So $t_{1}$ is regular and slim term. Now by Lemma 2.14 the value $t_{1}(\bar{p})$ contains the last state of the correct piece of the computation. But $t(\bar{p}) \neq w$, which proves that we have reached the halting state.

Theorem 2.17. Let $\mathcal{M}$ be a Minsky machine. The algebra $\mathbf{A}(\mathcal{M})$ has a nearunanimity term on the set $A(\mathcal{M}) \backslash\{r, w\}$ iff $\mathcal{M}$ halts.

Proof. Suppose that $t$ is a near-unanimity term on $A(\mathcal{M}) \backslash\{r, w\}$. Then $t$ is regular. Let $t^{\prime}$ be a term $p$-equivalent to $t$ and $p$-minimal. Then $t^{\prime}$ is not a variable; moreover, $t^{\prime}(\bar{p})=p$ implies that the leftmost operation symbol of $t^{\prime}$ (in prefix notation) is $M$. Now by Lemma 2.16, $\mathcal{M}$ halts. The other direction is proved in Lemma 2.13.

This finishes the proof of Theorem 2.2, as it is undecidable of a Minsky machine if it halts.

\section{TOWARDS THE DECIDABILITY OF A NU-TERM}

In this section we solve the NU-problem for special classes of algebras. We start with Rosenberg's primal algebra characterization (see $[13,11]$ ), which presents a natural framework for this. Clearly, a primal algebra has a ternary NU-term; and it is decidable if an algebra is primal. If the algebra is not primal, then its clone lies in one of the maximal clones described in Rosenberg's theorem. We solve the NUproblem in three classes of maximal clones (out of six), and present other partial results.

Rosenberg's characterization is in terms of six classes of finitary relations; a nontrivial finite algebra $\mathbf{A}$ is preprimal (its clone is a coatom in the lattice of clones) if and only if there is a relation $\varrho$ in one of the six classes such that the term functions of $\mathbf{A}$ are exactly the functions preserving the relation $\varrho$. Now we define these classes, following Quackenbush [11].

Definition 3.1. Let $A$ be a finite set.

A subset $\varrho \subseteq A^{2}$ is a partial order if it is reflexive $(\langle a, a\rangle \in \varrho$ for all $a \in A)$, antisymmetric $(\langle a, b\rangle,\langle b, a\rangle \in \varrho$ imply that $a=b)$, and transitive $(\langle a, b\rangle,\langle b, c\rangle \in \varrho$ imply that $\langle a, c\rangle \in \varrho$ ). We say that $b \in A$ is a zero (unit) of $\varrho \subseteq A^{2}$ if $\langle b, a\rangle \in \varrho$ $(\langle a, b\rangle \in \varrho)$ for all $a \in A$. Note that a partial order has at most one zero and at most one unit.

A subset $\varrho \subseteq A^{2}$ is a permutation if $\varrho=\{\langle a, \alpha(a)\rangle: a \in A\}$ where $\alpha: A \rightarrow A$ is a permutation on $A$. We say that the permutation $\varrho$ is prime if all cycles of $\alpha$ have the same length that is a prime number.

A subset $\varrho \subseteq A^{2}$ is an equivalence relation if $\varrho$ is reflexive, symmetric $(\langle a, b\rangle \in \varrho$ implies $\langle b, a\rangle \in \varrho)$, and transitive. An equivalence relation $\varrho$ is non-trivial if $\varrho \neq A^{2}$ and $\varrho \neq\{\langle a, a\rangle: a \in A\}$.

A subset $\varrho \subseteq A^{4}$ is affine if we can define an abelian group operation, + , on $A$ so that $\langle a, b, c, d\rangle \in \varrho$ if and only if $a+b=c+d$. An affine $\varrho$ is prime if $\langle A ;+\rangle$ is an elementary abelian $p$-group. 
A subset $\varrho \subseteq A^{h}$ (for $h \geq 1$ ) is totally symmetric if for every permutation $\alpha$ on $\{1, \ldots, h\},\left\langle a_{1}, \ldots, a_{h}\right\rangle \in \varrho$ if and only if $\left\langle a_{\alpha(1)}, \ldots, a_{\alpha(h)}\right\rangle \in \varrho$. Let $A_{h} \subseteq A^{h}$ be defined by

$$
A_{h}=\left\{\left\langle a_{1}, \ldots, a_{h}\right\rangle: a_{i}=a_{j} \text { for some } i \neq j\right\} .
$$

We say that $\varrho$ is totally reflexive if $A_{h} \subseteq \varrho$. Th center of $\varrho$ is the set of all $a \in A$ such that for all $a_{2}, \ldots, a_{h} \in A,\left\langle a, a_{2}, \ldots, a_{h}\right\rangle \in \varrho$. We say that $\varrho$ is central if it is totally symmetric, totally reflexive and has a center which is a non-empty, proper subset of $A$.

Let $h=\{0,1, \ldots, h-1\}$. For $1 \leq r \leq m$, let $\pi_{r}^{m}$ be the $r$ th projection of $h^{m}$ onto $h$. Define $\omega_{m}$ to be the $h$-ary relation on $h^{m}$ such that $\left\langle a_{1}, \ldots, a_{h}\right\rangle \in \omega_{m}$ if and only if for all $1 \leq r \leq m,\left\langle\pi_{r}^{m}\left(a_{1}\right), \ldots, \pi_{r}^{m}\left(a_{h}\right)\right\rangle \in h_{h}$ (where $h_{h}$ is defined by $(*)$ ). A subset $\varrho \subseteq A^{h}$ for $h \geq 3$ is $h$-regularly generated if for some $m \geq 1$ there is a surjection $\varphi: A \rightarrow h^{m}$ such that $\varrho=\varphi^{-1}\left(\omega_{m}\right)$; i.e., $\left\langle a_{1}, \ldots, a_{h}\right\rangle \in \varrho$ if and only if $\left\langle\varphi\left(a_{1}\right), \ldots, \varphi\left(a_{h}\right)\right\rangle \in \omega_{m}$. Clearly, if $\varrho$ is $h$-regularly generated, then $\varrho$ is totally reflexive and totally symmetric.

Theorem 3.2 (Rosenberg [13]). A finite non-trivial algebra $\mathbf{A}$ is preprimal if and only if there exists an h-ary relation $\varrho$ on A from the following classes

(1) the set of all partial orders with a zero and unit,

(2) the set of all prime permutations,

(3) the set of all non-trivial equivalence relations,

(4) the set of all prime affine relations,

(5) the set of all central relations,

(6) the set of all h-regularly generated relations,

such that the set of term functions of $\mathbf{A}$ is just the set of functions preserving $\varrho$.

First we show that the NU-term problem is decidable inside a maximal clone of class (1). We need the following lemma, which grew out of discussions with R. McKenzie.

Lemma 3.3. For a finite algebra $\mathbf{A}$ and a natural number $k$, it is decidable whether $\mathbf{A}$ has a near-unanimity term in which at most $k$ variables have repeated occurrences.

Proof. It is enough to effectively find a number $K$ so that if $\mathbf{A}$ has a NU-term in which at most $k$ variables have repeated occurrences, then it has a NU-term of depth at most $K$ with the same property.

Suppose we do have a near-unanimity term $t$, and its tree has a long branch $t=t_{0}, t_{1}, \ldots, t_{n}$. Here $t_{i}=g_{i}\left(t_{i+1},-, \ldots,-\right)$, where $g_{i}$ is a basic operation with variables permuted. Let $X$ be the tuple $x_{1}, x_{2}, \ldots, x_{k}$ of variables permitted to have repeated occurrences, and $Y$ be the tuple of remaining variables.

We find a long subsequence $\left\{s_{j}\right\}$ of $\left\{t_{i}\right\}$, such that when all variables of $Y$ are replaced by one new variable $z$, then $s_{j}(X ; z)=s_{l}(X ; z)$ for all $j$ and $l$. We can also assume that $B\left(s_{j}\right)=B\left(s_{l}\right)$ for all $j$ and $l$, where $B(s(X ; Y))$ is the set of all term operations $b(x, z)$ of $\mathbf{A}$ arising from the term $s(X ; Y)$ by choosing some variable among $Y$, replacing it by $z$, and then replacing all other variables of $Y$ and $X$ by $x$. Also, we can assume that $s_{j}(x, \ldots, x)=s_{l}(x, \ldots, x)$.

Now we claim that if we create a new term $t^{\prime}$ by replacing the explicit occurrence of $s_{1}$ in $t$ (i.e., at $t_{i}=g_{i}\left(s_{1},-, \ldots,-\right)$, where $\left.s_{1}=t_{i+1}\right)$ by $s_{2}$, then this shorter term $t^{\prime}$ is also a near-unanimity term. 
Indeed, in each near-unanimous assignment in which the minority variable is from $X$, the terms $s_{1}$ and $s_{2}$ behave the same. If the minority variable is from $Y$, then it has exactly one occurrence. If this occurrence is inside of $s_{1}$, then we use that fact that $B\left(s_{1}\right)=B\left(s_{2}\right)$. If it is outside, then we use that fact that $s_{1}(x, \ldots, x)=s_{2}(x, \ldots, x)$.

Corollary 3.4. Given a finite algebra $\mathbf{A}$ whose clone lies in a maximal clone of class (1). Then it is decidable if $\mathbf{A}$ has a near-unanimity term.

Proof. We will prove that if $\mathbf{A}$ has a NU-term, then it has a NU-term in which no variable has multiple occurrences. By the previous lemma this is enough.

Assume that $t\left(x_{1}, \ldots, x_{n}\right)$ is a NU-term of $\mathbf{A}$. Put

$$
t^{\prime}\left(y_{11}, \ldots, y_{1 m_{1}}, y_{21}, \ldots, y_{n m_{n}}\right)
$$

the term obtained from $t$ by replacing all occurrences of each variable $x_{i}$ by distinct variables $y_{i j}$. We claim that $t^{\prime}$ is also a NU-term. Let $\leq$ be a compatible partial order on $\mathbf{A}$ with a zero element $0 \in A$ and a unit element $1 \in A$. Take elements $a, b \in A$, and consider the near-unanimous assignment $t^{\prime}(a, \ldots, a, b, a, \ldots, a)$ where $y_{i j}=b$ for some $i$ and $j$. Since $\leq$ is compatible with $t^{\prime}$,

$$
\begin{aligned}
t^{\prime}(a, \ldots, a, b, a, \ldots, a) & \leq t^{\prime}(a, \ldots, a, 1, \ldots, 1, a, \ldots, a) \\
& =t(a, \ldots, a, 1, a, \ldots, a)=a,
\end{aligned}
$$

where $y_{i k}=1$ for all $k$, and $x_{i}=1$. On the other hand, $a \leq t^{\prime}(a, \ldots, a, b, a, \ldots, a)$ by a similar argument. Therefore

$$
t^{\prime}(a, \ldots, a, b, a, \ldots, a)=a
$$

for all $a, b \in A$ and $i, j$.

Now we show that no NU-term can exist in the maximal clones of class (4) and (6), so the problem is decidable in these cases. We call an algebra $\mathbf{A}$ affine if it has a compatible affine relation.

Proposition 3.5. No finite affine algebra has a near-unanimity term. In particular, a finite algebra A whose clone lies in a maximal clone of class (4), has no near-unanimity term.

Proof. Assume the contrary, that there exists a NU-term $t\left(x_{1}, \ldots, x_{n}\right)$ of $\mathbf{A}$. Let $0 \in A$ be the zero element of the abelian group $\langle A ;+\rangle$. Fix another element $a \neq 0$ of $A$. For $0 \leq k \leq n$, let $\bar{a}^{k}$ be the vector $\langle a, \ldots, a, 0, \ldots, 0\rangle \in A^{n}$ with $k$-many $a$ entries. We show by induction that $t\left(\bar{a}^{k}\right)=0$, which is a contradiction for $k=n$. The base of the induction, $k=0$, is true, since $t$ is a NU-term. For the induction step

$$
\begin{array}{ll}
t(a, \ldots, a, 0,0, \ldots, 0)=0, & \text { by the induction hypothesis, } \\
t(0, \ldots, 0, a, 0, \ldots, 0)=0, & \text { by the NU-term } t \\
t(0, \ldots, 0,0,0, \ldots, 0)=0, & \text { by the NU-term } t, \text { and } \\
t(a, \ldots, a, a, 0, \ldots, 0)=\mathrm{b}, & \text { for some } b \in A .
\end{array}
$$

On the left hand side all columns are in the relation $x+y=z+u$. Since this relation is preserved by $t, 0+0=0+b$, that is, $b=0$.

Proposition 3.6. A finite algebra $\mathbf{A}$ whose clone lies in a maximal clone of class (6), has no near-unanimity term. 
Proof. Let $h, m$ be natural numbers, $\varphi: A \rightarrow h^{m}$ be a surjection, and $\varrho \subseteq A^{h}$ be a relation as described in the Definition 3.1 under class (6). Assume that there exists a NU-term $t\left(x_{1}, \ldots, x_{n}\right)$ of $\mathbf{A}$ which preserves $\varrho$. We want to get a contradiction.

Recall that $h=\{0,1, \ldots, h-1\}$ and $h \geq 3$. Since $\varphi$ is surjective, there exist $a_{0}, \ldots, a_{h-1} \in A$ such that $\pi_{1}^{m}\left(\varphi\left(a_{i}\right)\right)=i$ for all $0 \leq i<h$. For $0 \leq k \leq n$ put $\bar{b}^{k}=\left\langle a_{0}, \ldots, a_{0}, a_{1}, \ldots, a_{1}\right\rangle \in A^{n}$ with $k$ many $a_{0}$ entries. We will prove by induction that $\pi_{1}^{m}\left(\varphi\left(t\left(\bar{b}^{k}\right)\right)\right) \neq 0$ for all $0 \leq k \leq n$. For $k=0$ this is true by definition.

For the induction step assume that the claim is true for $k$. Put $j=\pi_{1}^{m}\left(\varphi\left(t\left(\bar{b}^{k}\right)\right)\right)$. By the induction hypothesis, $j \neq 0$. Consider the following tuples of $A^{n}$

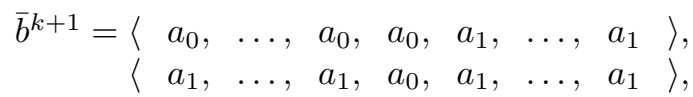

$$
\begin{aligned}
& \bar{b}^{k}=\begin{array}{c}
\left\langle a_{j-1}, \ldots, a_{j-1}, a_{0}, a_{j-1}, \ldots, a_{j-1}\right\rangle, \\
\left\langle a_{0}, \ldots, a_{0}, a_{1}, a_{1}, \ldots, a_{1}\right\rangle, \\
\left\langle a_{j+1}, \ldots, a_{j+1}, a_{0}, a_{j+1}, \ldots, a_{j+1}\right\rangle,
\end{array} \\
& \left\langle a_{h-1}, \ldots, a_{h-1}, a_{0}, a_{h-1}, \ldots, a_{h-1}\right\rangle,
\end{aligned}
$$

where the $i$ th row $(i \neq 0, j)$ is the near-unanimous $a_{i}$ tuple with $a_{0}$ at the $k+1$-th coordinate. Notice that each column has a repeated entry. Indeed, for the $k+1$-th column it is $a_{0}$, and for all other columns it is either $a_{0}$ or $a_{1}$ from the rows $\bar{b}^{k+1}$ and $\bar{b}^{k}$. This means that each column is in the relation $\varrho$. Therefore, by applying $t$,

$$
\left\langle t\left(\bar{b}^{k+1}\right), a_{1}, \ldots, a_{j-1}, t\left(\bar{b}^{k}\right), a_{j+1}, \ldots, a_{h-1}\right\rangle \in \varrho .
$$

Denote this tuple by $\bar{c}$. By the definition of $\varrho, \varphi(\bar{c}) \in \omega_{m}$. Then by the definition of $\omega_{m}, \pi_{1}^{m}(\varphi(\bar{c})) \in h_{h}$. But we can calculate this tuple,

$$
\pi_{1}^{m}(\varphi(\bar{c}))=\left\langle\pi_{1}^{m}\left(\varphi\left(t\left(\bar{b}^{k+1}\right)\right)\right), 1, \ldots, j-1, j, j+1, \ldots, h-1\right\rangle .
$$

By the definition of $h_{h}$, this tuple must have a repetition, thus $\pi_{1}^{m}\left(\varphi\left(t\left(\bar{b}^{k+1}\right)\right)\right) \neq 0$. This completes the proof of the induction step.

We have shown that $\pi_{1}^{m}\left(\varphi\left(t\left(\bar{b}^{n}\right)\right)\right) \neq 0$. On the other hand,

$$
\pi_{1}^{m}\left(\varphi\left(t\left(\bar{b}^{n}\right)\right)\right)=\pi_{1}^{m}\left(\varphi\left(t\left(a_{0}, \ldots, a_{0}\right)\right)\right)=\pi_{1}^{m}\left(\varphi\left(a_{0}\right)\right)=0,
$$

which is a contradiction.

In the rest of this section we focus on the case when the finite algebra in question is idempotent. As the first step we reduce the problem to simple algebras.

Definition 3.7. An algebra $\mathbf{A}$ is idempotent if $f(x, \ldots, x)=x$ for each basic operation $f$. Note that $\mathbf{A}$ cannot have constants, by definition, if $|A|>1$.

Lemma 3.8. The existence of a near-unanimity term for idempotent algebras is decidable if and only if it is decidable for simple idempotent algebras.

In order to prove this result we need the following definition and lemma which describe a way to compose NU-terms.

Definition 3.9. Let $s\left(x_{1}, \ldots, x_{n}\right)$ and $t\left(y_{1}, \ldots, y_{m}\right)$ be terms in $n$ and $m$ variables, respectively. Their star product $s \star t$ is a term in $n m$ variables defined as

$$
(s \star t)\left(z_{11}, \ldots, z_{n m}\right)=s\left(t\left(z_{11}, \ldots, z_{1 m}\right), \ldots, t\left(z_{n 1}, \ldots, z_{n m}\right)\right) .
$$


Lemma 3.10. Let $\mathbf{A}$ and $\mathbf{B}$ be similar idempotent algebras. If $s$ and $t$ are nearunanimity terms of $\mathbf{A}$ and $\mathbf{B}$, respectively, then $s \star t$ is a near-unanimity term of both $\mathbf{A}$ and $\mathbf{B}$.

Proof. First we prove the claim for A. Let $a, b \in A$, and put $c=(s \star t)(a, b, \ldots, b)$. We want to show that $c=b$. Notice that this is enough, as we did not assume any ordering of the variables of $s$ and $t$. By definition,

$$
c=s(t(a, b \ldots, b), t(b, \ldots, b), \ldots, t(b, \ldots, b)) .
$$

Since $\mathbf{A}$ is idempotent, $t(b, \ldots, b)=b$, and $c=s(t(a, b, \ldots, b), b, \ldots, b)$. As $s$ is a NU-term, we conclude that $c=b$. The proof for $\mathbf{B}$ is similar.

Proof of Lemma 3.8. One direction is trivial. For the other direction assume that the problem is decidable for simple idempotent algebras, and let $\mathbf{A}$ be a finite idempotent algebra, which is not simple. The decision procedure we present is recursive; we assume that for all algebras of cardinality less than of $\mathbf{A}$ we can decide the problem.

Let $\vartheta$ be a nontrivial congruence of $\mathbf{A}$, and $B$ be a congruence block of $\vartheta$. We claim that $B$ is a subuniverse of $\mathbf{A}$. Indeed, for each basic operation $f$ and elements $b_{1}, \ldots, b_{k} \in B, f\left(b_{1}, \ldots, b_{k}\right) \vartheta f\left(b_{1}, \ldots, b_{1}\right)=b_{1}$. Note that, by Definition 3.7, $f$ cannot be a constant.

Denote by $\mathbf{B}$ the subalgebra of $\mathbf{A}$ on the set $B$. If $\mathbf{A}$ has a NU-term, then the same term is a NU-term for $\mathbf{B}$. Similarly, the same term is a NU-term for $\mathbf{A} / \vartheta$. Therefore a necessary condition for $\mathbf{A}$ to have a NU-term is that each proper subalgebra and proper homomorphic image of $\mathbf{A}$ have a NU-term. We will show that this condition is sufficient, as well.

Let $t_{1}, \ldots, t_{n}$ be NU-terms on the nontrivial congruence blocks of $\vartheta$, respectively, and $s$ be a NU-term on $\mathbf{A} / \vartheta$. By Lemma 3.10, the term $t=t_{1} \star\left(t_{2} \star\left(\ldots\left(t_{n-1} \star\right.\right.\right.$ $\left.\left.t_{n}\right) \ldots\right)$ ) is a NU-term on each congruence block of $\vartheta$. We claim that $t \star s$ is a NU-term on A. Take $a, b \in A$. Since $s$ is idempotent on $A$ and a NU-term of $\mathbf{A} / \vartheta$,

$$
(t \star s)(a, \ldots, a, b, a, \ldots, a)=t\left(a, \ldots, a, b^{\prime}, a, \ldots, a\right)=a
$$

for some element $b^{\prime}=s(a, \ldots, a, b, a, \ldots, a) \vartheta a$.

We call an algebra A strictly simple if it is simple and has no non-trivial subalgebras. By a non-trivial subalgebra we mean a proper subalgebra having at least two elements.

Theorem 3.11 (Á. Szendrei $[14,15]$ ). Let A be a finite idempotent strictly simple algebra. Then the clone of $\mathbf{A}$ is one of the following clones.

(1) $|A|=2$ and Clo $\mathbf{A}$ is the trivial clone [id].

For a vector space $\mathbf{V}$ denote by End $\mathbf{V}$ the ring of endomorphisms of $\mathbf{V}$, and by (End $\mathbf{V}) \mathbf{V}$ the left module over End $\mathbf{V}$.

(2) A finite dimensional vector space $\mathbf{V}=\langle A ;+, K\rangle$ over a finite field $K$ can be defined on $A$, and Clo $\mathbf{A}$ is the clone $\mathrm{Clo}_{\mathrm{id}}((\mathrm{End} \mathbf{V}) \mathbf{V})$ of idempotent operations of $($ End $\mathbf{V}) \mathbf{V}$.

For a permutation group $G$ on $A$ let $\mathcal{R}_{\text {id }}(G)$ denote the clone of all idempotent operations $f$ on $A$ such that $f$ admits each member of $G$ as an automorphism. 
(3) Clo $\mathbf{A}=\mathcal{R}_{\mathrm{id}}(G)$ for some permutation group $G$ on $A$ such that every nonidentity member of $G$ has at most one fixed point.

Let $0 \in A$ be some fixed element. For $k \geq 2$ put

$\chi_{k}^{0}=\left\{\left\langle a_{1}, \ldots, a_{k}\right\rangle \in A^{k}: a_{i}=0\right.$ for at least one $\left.i, 1 \leq i \leq k\right\}$.

Denote by $\mathcal{F}_{k}^{0}$ the clone of all operations on $A$ preserving the relation $\chi_{k}^{0}$. Furthermore, put $\mathcal{F}_{\omega}^{0}=\bigcap_{k=2}^{\infty} \mathcal{F}_{k}^{0}$.

(4) Clo $\mathbf{A}=\mathcal{R}_{\mathrm{id}}(G) \cap \mathcal{F}_{k}^{0}$ for some $k(2 \leq k \leq \omega)$, some element $0 \in A$, and some permutation group $G$ on $A$ such that 0 is the unique fixed point of every non-identity member of $G$.

For a relation $\varrho$ on $A$ let $\mathcal{P}_{\varrho}$ denote the clone of operations on $A$ preserving $\varrho$.

(5) $|A|=2$ and Clo $\mathbf{A}=\mathcal{R}_{\text {id }}(G) \cap \mathcal{P}_{\leq}$for some permutation group $G$ on $A$; or $|A|=2$ and $\operatorname{Clo} \mathbf{A}=\mathcal{R}_{\text {id }}(\{\operatorname{id}\}) \cap \mathcal{P}_{\leq} \cap \mathcal{F}_{k}^{0}$ for some $k(2 \leq k \leq \omega)$ and some element $0 \in A$.

(6) $|A|=2$ and $\mathrm{Clo} \mathbf{A}$ is the clone $[\mathrm{V}]$ generated by the join operation; or $|A|=2$ and Clo $\mathbf{A}$ is the clone $[\wedge$ ] generated by the meet operation.

Proposition 3.12. The near-unanimity problem for idempotent, strictly simple algebras is decidable.

Proof. Let $\mathbf{A}$ be a idempotent, strictly simple algebra. We will use classification of Theorem 3.11 in the following decision procedure.

Assume that $\mathbf{A}$ has a compatible partial order relation with zero and unit. Clearly, this condition is decidable. Then by Corollary 3.4 the NU-problem is decidable. This handles the cases (1), (5) and (6) of Theorem 3.11.

Recall that the algebra $\mathbf{A}$ is called affine if it has a compatible affine relation. This is also a decidable property of $\mathbf{A}$. In Proposition 3.5 we have seen that if $\mathbf{A}$ is affine, then it has no NU-term. This handles case (2) of Theorem 3.11, because in that case $\mathrm{Clo} \mathbf{A}$ has a compatible affine relation.

If neither of the previous two conditions hold, then by Theorem 3.11 we know that Clo $\mathbf{A}$ is of type (3) or (4). In the rest of the proof we will show that the NU-problem is decidable even in these two cases.

Claim 1. Assume that Clo $\mathbf{A}=\mathcal{R}_{\text {id }}(G)$ as described in case (3) of Theorem 3.11. Then $\mathbf{A}$ has a ternary $N U$-term.

Consider the function $f: A^{3} \rightarrow A$, defined as

$$
f(a, b, c)= \begin{cases}\operatorname{maj}(a, b, c) & \text { if the majority exists, } \\ a & \text { otherwise. }\end{cases}
$$

Clearly, $f$ is a NU-term and admits all permutations on $A$.

Claim 2. Assume that Clo $\mathbf{A}=\mathcal{R}_{\mathrm{id}}(G) \cap \mathcal{F}_{k}^{0}$ as described in case (4) of Theorem 3.11, and $k<\omega$. Then $\mathbf{A}$ has a $N U$-term.

Consider the function $f: A^{k+1} \rightarrow A$, defined as

$$
f\left(a_{1}, \ldots, a_{k+1}\right)= \begin{cases}0 & \text { if } a_{i}=a_{j}=0 \text { for some } i \neq j, \\ \operatorname{maj}\left(a_{1}, \ldots, a_{k+1}\right) & \text { else if the majority exists } \\ a_{1} & \text { otherwise. }\end{cases}
$$


Clearly, $f$ is a NU-term. By the description of case (4), the element 0 is a fixed point of every member of $G$. Therefore $f \in R_{\text {id }}(G)$. To show that $f \in \mathcal{F}_{k}^{0}$, take $\bar{a}^{1}, \ldots, \bar{a}^{k+1} \in \chi_{k}^{0}$. By the Pigeon Hole Principle, there exist $i, i^{\prime}\left(1 \leq i, i^{\prime} \leq k+1\right)$ and $j(1 \leq j \leq k)$ such that $a_{j}^{i}=a_{j}^{i^{\prime}}=0$. This shows that $f\left(a_{j}^{1}, \ldots, a_{j}^{k+1}\right)=0$, therefore

$$
\left\langle f\left(a_{1}^{1}, \ldots, a_{1}^{k+1}\right), \ldots, f\left(a_{k}^{1}, \ldots, a_{k}^{k+1}\right)\right\rangle \in \chi_{n}^{0} .
$$

Claim 3. If Clo $\mathbf{A} \subseteq \mathcal{F}_{\omega}^{0}$ for some $0 \in A$, then $\mathbf{A}$ has no $N U$-term.

Assume the contrary, that $f \in \mathcal{F}_{\omega}^{0}$ is an $n$-ary NU-term. Take an element $a \in A \backslash\{0\}$, and consider the tuples $\bar{a}^{i}=\langle a, \ldots, a, 0, a, \ldots, a\rangle \in A^{n}$ for $1 \leq i \leq n$ where $a_{i}^{i}=0$. Clearly, $\bar{a}^{i} \in \chi_{n}^{0}$, and

$$
\left\langle f\left(a_{1}^{1}, \ldots, a_{1}^{n}\right), \ldots, f\left(a_{n}^{1}, \ldots, a_{n}^{n}\right)\right\rangle=\langle a, \ldots, a\rangle \notin \chi_{n}^{0} .
$$

This shows that $f \notin \mathcal{F}_{n}^{0}$, which is a contradiction.

Claim 4. Fix an element $0 \in A$. Then $\mathcal{F}_{k}^{0} \supseteq \mathcal{F}_{k+1}^{0}$ for all $k \geq 2$.

Take a function $f: A^{n} \rightarrow A$ preserving $\chi_{k+1}^{0}$. To show that it preserves $\chi_{k}^{0}$, as well, take $\bar{a}^{1}, \ldots, \bar{a}^{n} \in \chi_{k}^{0}$. Put $\bar{b}^{i}=\left\langle\bar{a}^{i}, a_{k}^{i}\right\rangle=\left\langle a_{1}^{i}, \ldots, a_{k}^{i}, a_{k}^{i}\right\rangle$ for $1 \leq i \leq n$. Clearly, $\bar{b}^{i} \in \chi_{k+1}^{0}$. Since $f$ preserves $\chi_{k+1}^{0}$, the tuple

$$
\begin{aligned}
& \left\langle f\left(b_{1}^{1}, \ldots, b_{1}^{n}\right), \ldots, f\left(b_{k}^{1}, \ldots, b_{k}^{n}\right), f\left(b_{k+1}^{1}, \ldots, b_{k+1}^{n}\right)\right\rangle \\
= & \left\langle f\left(a_{1}^{1}, \ldots, a_{1}^{n}\right), \ldots, f\left(a_{k}^{1}, \ldots, a_{k}^{n}\right), f\left(a_{k}^{1}, \ldots, a_{k}^{n}\right)\right\rangle
\end{aligned}
$$

is in relation $\chi_{k+1}^{0}$. This means that $\left\langle f\left(a_{1}^{1}, \ldots, a_{1}^{n}\right), \ldots, f\left(a_{k}^{1}, \ldots, a_{k}^{n}\right)\right\rangle \in \chi_{k}^{0}$, which is what we wanted to show.

Claim 5. Let $f$ be an $n$-ary function on $A$, and $0 \in A$. If $f \in \mathcal{F}_{n}^{0}$, then $f \in \mathcal{F}_{k}^{0}$ for all $n \leq k \leq \omega$.

Fix $k$ such that $n \leq k<\omega$, and take $\bar{a}^{1}, \ldots, \bar{a}^{n} \in \chi_{k}^{0}$. By definition, there exists a "choice function" $\zeta:\{1, \ldots, n\} \rightarrow\{1, \ldots, k\}$ such that $a_{\zeta(i)}^{i}=0$ for all $1 \leq i \leq n$. Put $\bar{b}^{i}=\left\langle a_{\zeta(1)}^{i}, \ldots, a_{\zeta(n)}^{i}\right\rangle$ for $1 \leq i \leq n$. Since $b_{i}^{i}=0, \bar{b}^{i} \in \chi_{n}^{0}$. By our hypothesis,

$$
\left\langle f\left(b_{1}^{1}, \ldots, b_{1}^{n}\right), \ldots, f\left(b_{n}^{1}, \ldots, b_{n}^{n}\right)\right\rangle \in \chi_{n}^{0} .
$$

This means that $f\left(b_{j}^{1}, \ldots, b_{j}^{n}\right)=0$ for some $1 \leq j \leq n$, therefore $f\left(a_{\zeta(j)}^{1}, \ldots, a_{\zeta(j)}^{n}\right)=$ 0. Hence $f \in \mathcal{F}_{k}^{0}$. Finally, since $\mathcal{F}_{2}^{0} \supseteq \cdots \supseteq \mathcal{F}_{\omega}^{0}$ and $f \in \mathcal{F}_{k}^{0}$ for all $n \leq k<\omega$, $f \in F_{\omega}^{0}$.

Claim 6. Assume that Clo A is of type (3) or (4) as described in Theorem 3.11. Then it is decidable if $\mathbf{A}$ has a NU-term.

First we check if $\mathbf{A}$ has a ternary NU-term. If it does, then we are done. Assume that $\mathbf{A}$ has no ternary NU-term. Then by Claim 1, Clo A is of type (4). Moreover, by Claims 2 and 3, $\mathbf{A}$ has no NU-term if and only if Clo $\mathbf{A} \subseteq \mathcal{F}_{\omega}^{0}$ for some $0 \in A$.

Now we show that, given $0 \in A$, it is decidable if Clo $\mathbf{A} \subseteq \mathcal{F}_{\omega}^{0}$. Take a basic $n$-ary operation $f$ of $\mathbf{A}$. Clearly, we can decide if $f \in \mathcal{F}_{n}^{0}$. If $f \in \mathcal{F}_{n}^{0}$, then $f \in \mathcal{F}_{\omega}^{0}$, otherwise $f \notin \mathcal{F}_{\omega}^{0}$. So, Clo $\mathbf{A} \subseteq \mathcal{F}_{\omega}^{0}$ if and only if $f \in \mathcal{F}_{n}^{0}$ for all basic operations $f\left(x_{1}, \ldots, x_{n}\right)$. 


\section{REFERENCES}

[1] S. Burris and H. P. Sankappanavar, A course in universal algebra. Graduate Texts in Mathematics 78, Springer-Verlag, New York, 1981.

[2] B. A. Davey, Duality theory on ten dollars a day. Algebras and Orders (Montreal, 1991), NATO Advanced Study Institute Series, Series C, 389, 71-111.

[3] B. A. Davey, L. Heindorf and R. McKenzie, Near unanimity: an obstacle to general duality theory. Algebra Universalis, 33 (1995), 428-439.

[4] B. A. Davey and H. Werner, Dualities and equivalences for varieties of algebras. Contributions to lattice theory (Szeged, 1980), Colloq. Math. Soc. János Bolyai, 33 (1983), 101-275.

[5] J. Ježek and M. Maróti, Membership problems for finite entropic groupoids. (manuscript).

[6] O. G. Kharlampovich and M. V. Sapir, Algorithmic problems in varieties. Int. Journal of Algebra and Comp., 5 (1995), 379-602.

[7] R. McKenzie, Is the presence of a nu-term a decidable property of a finite algebra? October 15, 1997 (manuscript).

[8] R. McKenzie, G. McNulty and W. Taylor, Algebras, Lattices, Varieties, Volume I. Wadsworth \& Brooks/Cole, Monterey, CA, 1987.

[9] M. L. Minsky, Recursive unsolvability of Post's problem of "tag" and other topics in the theory of Turing Machines. Ann. Math., 74 (1961), 437-455.

[10] M. L. Minsky, Computations: finite and infinite machines. Prentice-Hall, Englewood Cliffs, N.J., 1967.

[11] R. W. Quackenbush, A new proof of Rosenberg's primal algebra characterization theorem. Finite algebra and multiple-valued logic (Szeged, 1979), Colloq. Math. Soc. János Bolyai, 28 (1981), 603-634.

[12] M. O. Rabin and D. Scott, Finite automata and their decision problems. IBM Journal of Res. and Devel., 3(2) (1969), 114-125.

[13] I. Rosenberg, Über die funktionale Vollständigkeit in den mehrwertigen Logiken. Rozpravy Československe Akad. Věd., Ser. Math. Nat. Sci., 80 (1970), 3-93.

[14] Á. Szendrei, Idempotent algebras with restrictions on subalgebras. Acta Sci. Math. (Szeged) 51 (1987), 251-268.

[15] Á. Szendrei, Term minimal algebras. Algebra Universalis, 32 (1994), 439-477.

Bolyai Institute, University of Szeged, Aradi Vértanúk tere 1., 6720 Szeged, HunGARY

E-mail address: mmaroti@math.u-szeged.hu 